\title{
SUFFICIENT CONDITIONS FOR CLOSE-TO-CONVEXITY OF AN ANALYTIC FUNCTION IN THE UNIT DISC
}

\author{
K. S. PADMANABHAN*, RAJALAKSHMI RAJAGOPAL AND C. SELVARAJ
}

\begin{abstract}
Recently C. Ramesha and others have derived a sufficient condition for functions analytic in the unit disc to be starlike. Subsequently M. Nunokowa and others have improved the earlier results of C. Ramesha and others. In this paper we generalise the results of C. Ramesha and others in a different direction and obtain sufficient conditions for $f(z)$ to be close-to-convex in the unit disc $E$.
\end{abstract}

\section{Introduction}

Let $A$ denote the class of functions, which are analytic in the unit disc $E$ and normalized by the conditions $f(0)=0 ; f^{\prime}(0)=1$. Let $S$ denote the subclass of $A$, containing univalent functions. Let $S^{*}$ denote the subclass of $S$ containing functions which are starlike with respect to the origin. We say that $f \in S^{*}(\alpha), 0 \leq \alpha<1$, if and only if $\operatorname{Re} z f^{\prime}(z) / f(z)>\alpha, z \in E . S^{*}(0)=S^{*}$ is the class of starlike functions in $E . f \in A$ is said to be close-to-convex in $E$, if there exists a function $g \in S^{*}$ and a real number $\eta,|\eta|<\frac{\pi}{2}$, such that $\operatorname{Re}\left\{e^{i \eta} z f^{\prime}(z) / g(z)\right\}>0, z \in E$. The class of close-to-convex is denoted by $C$. Sufficient conditions for functions to be starlike or convex have been investigated frequently by different authors.

Sufficient criteria for convexity involving higher order derivatives of $f$ are investigated by $H$. Silverman [5]. More recently $H$. Silverman [6] considered the class $G_{b}$ consisting of normalized functions $f$ defined by $G_{b}=\left\{f:\left|\frac{1+z f^{\prime \prime}(z) / f^{\prime}(z)}{z f^{\prime}(z) / f(z)}-1\right|<b, z \in E\right\}$ and found sharp values of $b$ for which $G_{b} \subset S^{*}(\alpha), \frac{1}{2} \leq \alpha<1$ and also found the values of $b$ for which $G_{b} \subset K$, the class of convex functions in $E$. We note that Theorems 1 and 2 proved by him in [6] also holds for the meromorphic case with same type of argument. Recently C. Ramesha et al. [3] derived sufficient conditions for $f \in A$ to lie in $S^{*}$.

Subsequently M. Nunokowa and others [2] improved their result. In this paper we generalise the result in [3], in a different direction and obtain sufficient conditions for $f(z)$ to be in $C$. We require the following lemmas to prove our main results.

Received May 7, 2001; revised May 30, 2002.

2000 Mathematics Subject Classification. Primary 30C45, secondary 30 C50.

Key words and phrases. Close-to-Convex, Starlike.

* Deceased on June 2, 2002. 
Lemma 1.[1] Let $\omega$ be regular in the unit disc $E$ with $\omega(0)=0$. If there exists a point $z_{0} \in E$ such that $\max _{|z|=\left|z_{0}\right|}|\omega(z)|=\left|\omega\left(z_{0}\right)\right|$, then $z_{0} \omega^{\prime}\left(z_{0}\right)=k \omega\left(z_{0}\right)$ for some constant $k \geq 1$.

The following result is a special case of Theorem 4 [4] which we quote as

Lemma 2. Let $p(z)$ be analytic in $E$ with $p(0)=1$ and $p(z) \not \equiv 1$. If $z_{0}=r_{0} e^{i \theta_{0}}(0<$ $\left.r_{0}<1\right)$ and $\operatorname{Re} p\left(z_{0}\right)=\min _{|z|<r_{0}} \operatorname{Re} p(z)$, then $z_{0} p^{\prime}\left(z_{0}\right)$ is real and

$$
z_{0} p^{\prime}\left(z_{0}\right) \leq-\frac{1}{2}\left\{1+\left(\operatorname{Im} p\left(z_{0}\right)\right)^{2}\right\} \quad \text { if } \quad \operatorname{Re} p\left(z_{0}\right)=0 .
$$

\section{Main Results}

Theorem 1. Let $M_{b}=\left\{f:\left|\frac{1+z f^{\prime \prime}(z) / f^{\prime}(z)}{z f^{\prime}(z) / f(z)}-1\right|<b, \quad z \in E \backslash 0\right\}$ where $f$ is given by $f(z)=\frac{1}{z}+a_{0}+a_{1} z+a_{2} z^{2}+\cdots$ with the condition $f(z) f^{\prime}(z) \neq 0$ in $z \in E \backslash 0$. If $0<b \leq 1$ and $f \in M_{b}$, then $f$ is meromorphic starlike of order $2 /(1+\sqrt{1+8 b})$.

Theorem 2. Let $f \in A$ with $f(z) / z \neq 0, z \in E$ and satisfy the condition

$$
\operatorname{Re}\left\{\alpha z^{2} f^{\prime}(z) / g(z)+z f^{\prime}(z) / g(z)\right\}>-\alpha / 2
$$

where $g \in S^{*}$ and

$$
\operatorname{Re}\left(g^{\prime}(z) / f^{\prime}(z)\right) \geq-1 / 2
$$

Then $f \in C$ for any $\alpha \geq 0$.

Theorem 3. Let $f \in A$ with $f(z) / z \neq 0$, and let $f$ satisfy the condition

$$
\operatorname{Re}\left\{\alpha z^{2} \frac{f^{\prime \prime}(z)}{g(z)}+\frac{z f^{\prime}(z)}{g(z)}\right\}>-\frac{\alpha}{2}\left\{1-\left|\operatorname{Im} \frac{z f^{\prime}(z)}{g(z)}\right|\right\}^{2}
$$

where $g \in S^{*}$ and $\left|\operatorname{Im} \frac{z g^{\prime}(z)}{g(z)}\right|<1$ for $z \in E$. Then $\operatorname{Re}\left\{\frac{z f^{\prime}(z)}{g(z)}\right\}>0$ for $z \in E$, that is $f \in C$.

Theorem 4. For $0<\beta<\alpha, C(\alpha) \subset C(\beta)$ where $C(\alpha)$ is the class of function $f$ satisfying the condition of Theorem 1.

Proof of Theorem 1. The details of proof of Theorem 1 are omitted, since they are exactly same as the proof of Theorem 1 in [6].

Proof of Theorem 2. Let $\frac{z f^{\prime}(z)}{g(z)}=\frac{1+\omega(z)}{1-\omega(z)}$, where $\omega(z)$ is analytic in $E$, with $\omega(0)=0$ and $\omega(z) \neq 1$. Then

$$
\alpha z^{2} \frac{f^{\prime \prime}(z)}{g(z)}+z \frac{f^{\prime}(z)}{g(z)}=\alpha\left\{\frac{2 z \omega^{\prime}(z)}{(1-\omega(z))^{2}}+\left\{\frac{1+\omega(z)}{1-\omega(z)}\right\}^{2} \frac{g^{\prime}(z)}{f^{\prime}(z)}\right\}+(1-\alpha) \frac{1+\omega(z)}{1-\omega(z)}
$$


We claim that $|\omega(z)|<1$. If there exists a $z_{0}$ in $E$ such that $\left|\omega\left(z_{0}\right)\right|=1$, then by Lemma 1 , we have $z_{0} \omega^{\prime}\left(z_{0}\right)=k \omega\left(z_{0}\right)$ for some $k \geq 1$.

Let $\omega\left(z_{0}\right)=e^{i \theta_{0}}$ for some $\theta_{0}$ with $0 \leq \theta_{0} \leq 2 \pi$. Using this in (4), on simplification, we obtain

$$
\operatorname{Re} \alpha z_{0}^{2} \frac{f^{\prime \prime}\left(z_{0}\right)}{g\left(z_{0}\right)}+z_{0} \frac{f^{\prime}\left(z_{0}\right)}{g\left(z_{0}\right)}=\frac{-\alpha}{2}\left\{\frac{k+2 \cos ^{2}\left(\frac{\theta_{0}}{2}\right) \operatorname{Re}\left(\frac{g^{\prime}\left(z_{0}\right)}{f^{\prime}\left(z_{0}\right)}\right)}{\sin ^{2}\left(\frac{\theta_{0}}{2}\right)}\right\} \leq-\frac{\alpha}{2}
$$

which follows since $\operatorname{Re}\left(\frac{g^{\prime}\left(z_{0}\right)}{f^{\prime}\left(z_{0}\right)}\right) \geq \frac{-1}{2}$.

Thus we get a contradiction to (1) proving that $|\omega(z)|<1$ for all $z \in E$ and $f \in C$.

Proof of Theorem 3. Setting $p(z)=\frac{z f^{\prime}(z)}{g(z)}$, we obtain after simplification

$$
\operatorname{Re} \alpha z^{2} \frac{f^{\prime \prime}(z)}{g(z)}+\frac{z f^{\prime}(z)}{g(z)}=\alpha z p^{\prime \prime}(z)+\alpha z p(z) \frac{g^{\prime}(z)}{g(z)}+(1-\alpha) p(z) .
$$

If $\operatorname{Re} p(z)>0$, for $|z|<\left|z_{0}\right|<1$ and $\operatorname{Re} p\left(z_{0}\right)=0$, then by Lemma (2), we have

$$
\begin{aligned}
\operatorname{Re}\left\{\alpha z_{0}^{2} \frac{f^{\prime \prime}\left(z_{0}\right)}{g\left(z_{0}\right)}+\frac{f^{\prime}\left(z_{0}\right)}{g\left(z_{0}\right)}\right\} & =\operatorname{Re}\left\{\alpha z_{0} p^{\prime}\left(z_{0}\right)+\alpha p\left(z_{0}\right) z_{0} \frac{g^{\prime}\left(z_{0}\right)}{g\left(z_{0}\right)}+(1-\alpha) p\left(z_{0}\right)\right\} \\
& \leq \frac{-\alpha}{2}\left[1+\left(\operatorname{Im} p\left(z_{0}\right)\right)^{2}\right]-\alpha \beta_{0} \gamma_{0}
\end{aligned}
$$

where $\beta_{0}=\operatorname{Im} z_{0} \frac{g^{\prime}\left(z_{0}\right)}{g\left(z_{0}\right)}, \gamma_{0}=\operatorname{Im} p\left(z_{0}\right)=\operatorname{Im} z_{0} \frac{f^{\prime}\left(z_{0}\right)}{g\left(z_{0}\right)}$

Right hand member of (5) becomes $\frac{-\alpha}{2}\left[1+\gamma_{0}^{2}+2 \gamma_{0} \beta_{0}\right]$ which is $<\frac{-\alpha}{2}\left(1-\left|\gamma_{0}^{2}\right|\right)$, which is contradiction to (3). Therefore $\operatorname{Re} p(z)>0$ for $f \in E$. That is, $f \in C$.

Proof of Theorem 4. $f \in C(\alpha)$ implies that $\alpha M+B>0$ where

$$
B=\operatorname{Re} \frac{z f^{\prime}(z)}{g(z)} \quad \text { and } \quad M=\operatorname{Re} z^{2} \frac{f^{\prime \prime}(z)}{g(z)}+\frac{1}{2}
$$

By Theorem 1, B>0 for $f \in C(\alpha)$. If (2) holds for some fixed $\alpha>0$ we claim that it is also true for all $\beta$ with $0<\beta<\alpha$. To see this we re-write (6) in the form $M+\frac{B}{\alpha}>0$.

Since for $0<\beta<\alpha$,

$$
M+\frac{B}{\beta}>M+\frac{B}{\alpha}>0 .
$$

From (7) we get $\beta M+B>0$ which implies that $f \in C(\beta)$.

\section{Acknowledgement}

The authors are grateful to the referee for his valuable suggestions. 


\section{References}

[1] I. S. Jack, Functions starlike and convex of order $\alpha$, J. London Math. Soc. 3(1971), 469 -474 .

[2] M. Nunokowa, S. Owa, S. K. Lee, M. Obradovic, M. K. Aouf, H. Saitoh, A. Ikeda and N. Koike, Sufficient conditions for starlikeness, Chinese J. Math. 24(1996), 265-271.

[3] C. Sampathkumar Ramesh and K. S. Padmanabhan, Sufficient conditions for starlikeness, Chinese J. Math. 23(1995), 167-171.

[4] S. Miller Sanford, and T. Mocanu Petru, Second order differential inequalities in the complex plane, J. Math. Anal. Appl. 65(1978), 289-305.

[5] Silverman, H., Higher order derivatives, Chinese J. Math. 23(1995), 189-191.

[6] Silverman, H., Convex and starlike criteria, Internat. J. Math. and Math. Sci. 22(1999), 75-79.

M73/2, $31^{\text {st }}$ Cross Street, Beasant Nagar, Chennai-600090.

Department of Mathematics, Loyola College, Chennai-600034.

Department of Mathematics, L. N. Government College, Ponneri-601204. 